\title{
Configuración y montaje de instrumento para la caracterización magnetoeléctrica de compuestos cerámicos multiferroicos
}

\author{
J. Camargo, M. Lere, M. Castro, y L. Ramajo* \\ Instituto de Investigaciones en Ciencia y Tecnología de Materiales, \\ Av. Colón 10850, Mar del Plata, Argentina. \\ * Dirección Postal: B7606BWV \\ TE: +54 223 6260633; FAX: +54 223 481-0046 \\ e-mail:lramajo@fi.mdp.edu.ar
}

Received 3 September 2019; accepted 14 May 2020

Este trabajo presenta la configuración y el montaje de un equipo empleado para cuantificar el coeficiente magnetoeléctrico dinámico ( $\alpha$ ) de compuestos cerámicos multiferroicos. En particular, se analizan las propiedades magnetoeléctricas de los compuestos de composición $\mathrm{Bi}_{0.5}\left(\mathrm{Na}_{0.8} \mathrm{~K}_{0.2}\right)_{0.5} \mathrm{TiO}_{3}-\mathrm{Ni}_{0.5} \mathrm{Co}_{0.5} \mathrm{Fe}_{2} \mathrm{O}_{4}$ (BNKT-NCF). En el método dinámico, la señal magnetoeléctrica (ME) se registra midiendo el potencial eléctrico a través de la muestra bajo un campo magnético continuo y variable en presencia de un campo magnético de CA. Los elementos de medición constan de un sistema de polarización (eléctrico y magnético), para polarizar la cerámica magnetoeléctrica, y un lock-in para generar la señal alterna y filtrar el ruido. Además, dada la baja señal ME, es necesario un blindaje adecuado y una sección fina de electrodos junto al amplificador tipo lock-in. El rendimiento de los dispositivos fabricados resulta satisfactorio para la medición dinámica del efecto ME para materiales magnetoeléctricos basados en cerámicos multiferroicos.

Descriptores: Instrumentación; coeficiente magnetoeléctrico; multiferroicos.

In the present work, the configuration and assembly of a device used to quantify the dynamic magnetoelectric coefficient $(\alpha)$ of multiferroic lead-free ceramic is analyzed. In particular, the magnetoelectric properties of $\mathrm{Bi}_{0.5}\left(\mathrm{Na}_{0.8} \mathrm{~K}_{0.2}\right)_{0.5} \mathrm{TiO}_{3}-\mathrm{Ni}_{0.5} \mathrm{Co}_{0.5} \mathrm{Fe}_{2} \mathrm{O}_{4}$ (BNKT-NCF) ceramics are studied. In the dynamic method, the magnetoelectric signal (ME) is recorded measuring the electrical potential across the sample under a DC magnetic field in the presence of an AC magnetic field. The measuring elements consist of a polarization system (electric and magnetic), to polarize the magnetoelectric ceramics, and a lock-in to generate the alternating signal and filter out the noise. Besides due to the low ME signal, adequate shielding and a thin electrode section are necessary close to the lock-in amplifier. The performance of the manufactured devices is satisfactory to perform the dynamic measurement of the ME effect for magnetoelectric materials based on multiferroic ceramics.

Keywords: Instrumentation; magnetoelectric coefficient; multiferroic materials.

PACS: $06 ; 78 ; 81$.

\section{Intruducción}

El efecto magnetoeléctrico es el fenómeno mediante el cual un campo magnético $(H)$ produce una polarización eléctrica, o un campo eléctrico $(E)$ origina una magnetización. Este efecto ha ganado gran atención debido a sus posibles aplicaciones en la nueva generación de detectores de campo magnético, sensores, transductores, así como para el avance en el almacenamiento magnético [1].

Materiales tales como $\mathrm{BiFeO}_{3}$ presentan propiedades magnetoeléctricas en forma intrínseca [2-3], aunque es posible combinar un material con propiedades piezoeléctricas con otro con propiedades magnetostrictivas con el objetivo de generar un compuesto magnetoeléctrico multiferroico [3-4]. En ambos casos, estos sistemas se caracterizan por poseer simultáneamente más de un tipo de ordenamiento magnético, eléctrico o elástico. En este sentido, es sabido que el orden magnético se encuentra estrechamente ligado a las interacciones entre dipolos magnéticos, las cuales se originan a partir del déficit de electrones en los orbitales superiores del átomo. Asimismo, el orden eléctrico es el resultado del ordenamiento de dipolos eléctricos locales, mientras que el orden elástico resulta del alineamiento de los desplazamientos atómicos debido a la deformación. Como se mencionó,
DOI: https://doi.org/10.31349/RevMexFis.66.462

la aparición simultánea del orden magnético y eléctrico es particularmente interesante, pues combina propiedades que podrían utilizarse para el almacenamiento, el procesamiento y la transmisión de información, debido a que permite que los campos magnéticos y eléctricos interactúen con el orden magnético y eléctrico [5].

En general, los métodos experimentales para medir el coeficiente magnetoeléctrico son los métodos estáticos, cuasi estáticos, dinámicos y dinámicos pulsados [6]. Para el método dinámico, la muestra se somete a la acción de un campo magnético alterno superpuesto en un campo magnético continuo y variable, que genera en los extremos del material una respuesta de voltaje (señal ME) que permite obtener, el valor indirectamente [7], con base a la Ec. (1). Este método ha tenido una gran recepción, ya que reduce los problemas de acumulación de carga en el borde de la muestra. Las ecuaciones básicas [8] necesarias para evaluar el coeficiente ME a partir del voltaje se describen a continuación. Ante un campo magnético continuo aplicado, el voltaje ME de salida en un material policristalino muestra un efecto de segundo orden que se puede cuantificar mediante:

$$
V \propto(\alpha H+\beta \cdot H \cdot H),
$$


donde $\alpha$ y $\beta$ son los coeficientes lineales y cuadráticos del componente ME de la muestra. Si un campo alterno $h_{0}$ se superpone a un campo continuo $H_{0}$, el campo efectivo es:

$$
H=H_{0}+h_{0} \cdot \sin (\omega t) \text {. }
$$

Si se emplea un amplificador lock-in es posible sintonizar una frecuencia establecida $\omega(\omega=2 \pi f)$ para medir la fem de salida y la salida ME se determina por:

$$
V \propto\left(\alpha \cdot H+\beta \cdot H_{0}\right)=\alpha^{*} \cdot\left(H_{0}\right) \cdot h_{0} .
$$

Este trabajo muestra la configuración de medida y los resultados experimentales de un equipo capaz de determinar el coeficiente $\mathrm{ME}$ de materiales cerámicos multiferroicos basados en compuestos de composición $\mathrm{Bi}_{0.5}\left(\mathrm{Na}_{0.8} \mathrm{~K}_{0.2}\right)_{0.5} \mathrm{TiO}_{3} /$ $\mathrm{Ni}_{0.5} \mathrm{Co}_{0.5} \mathrm{Fe}_{2} \mathrm{O}_{4}$ (BNKT-NCF) obtenidos por reacción en estado sólido.

\section{Experimental}

\subsection{Configuración}

La Fig. 1 muestra el diagrama esquemático del equipo empleado para medir los valores de coeficiente ME de muestras cerámicas magnetoeléctricas polarizadas. El equipo consta de un generador de campo magnético continuo (Dexing Magnet Model F2030) y otro alterno (fabricación propia). El primero consiste en un electroimán (núcleo de hierro) y una fuente de corriente continua que lo alimenta, mientras que el segundo se compone de una fuente de corriente alterna y una bobina de Helmholtz con núcleo de aire (diámetro $25 \mathrm{~mm}, 34$ vueltas). La bobina de Helmholtz permite una mayor zona de homogeneidad de campo magnético alterno para mejorar la sensibilidad de medida sobre la muestra en estudio. No obstante, fue necesario diseñar y caracterizar las bobinas

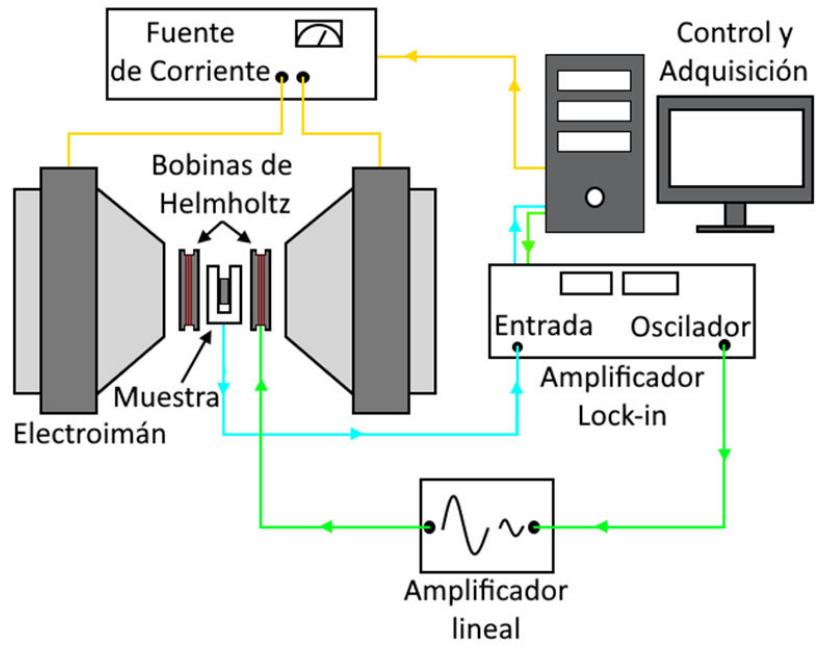

FIGURA 1. Diagrama en bloques de la configuración para medir la constante magnetoeléctrica de muestras cerámicas multiferroicas polarizadas. espacialmente para determinar la distribución de la densidad de flujo magnético, con la intención de establecer el valor y la región donde el campo es más intenso y homogéneo.

El generador de campo magnético alterno se compone de los siguientes conjuntos funcionales: un lock-in o generador de señales, un amplificador de potencia y unas bobinas de Helmholtz. Dado que el voltaje generado a través de la muestra es muy débil, lo que implica una respuesta magnetoeléctrica del orden de $1 \mathrm{mV} / \mathrm{cmOe}$, es necesario que las características del campo magnético generado por las bobinas de Helmholtz permanezcan constantes en el tiempo; es decir, que la densidad de flujo magnético, la frecuencia de campo y el tipo de onda sean muy estables.

\subsection{Preparación de las muestras}

El material compuesto se preparó mezclando los polvos cerámicos de ambos sistemas $\left(\mathrm{Ni}_{0.5} \mathrm{Co}_{0.5} \mathrm{Fe}_{2} \mathrm{O}_{4}\right.$ (NCF) y $\left.\mathrm{Bi}_{0.5}\left(\mathrm{Na}_{0.8} \mathrm{~K}_{0.2}\right)_{0.5} \mathrm{TiO}_{3}(\mathrm{BNKT})\right)$ previamente sintetizados por separado por reacción en estado sólido. Los reactivos utilizados para obtener la fase NCF fueron acetato de cobalto $\left(\mathrm{Co}\left(\mathrm{C}_{2} \mathrm{H}_{3} \mathrm{O}_{2}\right)_{2}\right.$, reactivo comercial), acetato de níquel $\left(\mathrm{Ni}\left(\mathrm{C}_{2} \mathrm{H}_{3} \mathrm{O}_{2}\right)_{2}\right.$, Aldrich $99 \%$, USA) y hematita $\left(\mathrm{Fe}_{2} \mathrm{O}_{3}\right.$, Mallinckrodt $97 \%$, USA), tal como se muestra a continuación:

$$
\begin{aligned}
& \mathrm{Co}\left(\mathrm{C}_{2} \mathrm{H}_{3} \mathrm{O}_{2}\right)_{2} \cdot 4 \mathrm{H}_{2} \mathrm{O}+\mathrm{Ni}\left(\mathrm{C}_{2} \mathrm{H}_{3} \mathrm{O}_{2}\right)_{2}+2 \mathrm{Fe}_{2} \mathrm{O}_{3} \cdot 4 \mathrm{H}_{2} \mathrm{O} \\
& +8 \mathrm{O}_{2} \rightarrow 2 \mathrm{Ni}_{0.5} \mathrm{Co}_{0.5} \mathrm{Fe}_{2} \mathrm{O}_{4}+8 \mathrm{CO}_{2}+14 \mathrm{H}_{2} \mathrm{O}
\end{aligned}
$$

Para obtener la fase BNKT se utilizaron $\mathrm{Bi}_{2} \mathrm{O}_{3}$ (Aldrich $99.8 \%$; USA), $\mathrm{Na}_{2} \mathrm{CO}_{3}$ (Aldrich 99.5\%; USA), $\mathrm{K}_{2} \mathrm{CO}_{3}$ (Aldrich $99.5 \%$; USA) y $\mathrm{TiO}_{2}$ (Aldrich $99.9 \%$; USA).

$$
\begin{aligned}
\mathrm{Bi}_{2} \mathrm{O}_{3} & +0.8 \mathrm{Na}_{2} \mathrm{CO}_{3}+0.2 \mathrm{~K}_{2} \mathrm{CO}_{3} \\
& +4 \mathrm{TiO}_{2} \rightarrow 4 \mathrm{Bi}_{0.5}\left(\mathrm{Na}_{0.8} \mathrm{~K}_{0.2}\right)_{0.5} \mathrm{TiO}_{3}+\mathrm{CO}_{2} .
\end{aligned}
$$

Los reactivos de ambas fases se mezclaron por separado durante 6 horas en medio alcohólico en un molino planetario (Fritsch, Pulverisette 7, $1450 \mathrm{rpm}$ ); luego, los polvos precursores de las fases BNKT y NCF se calcinaron por separado a 700 y $1050^{\circ} \mathrm{C}$, respectivamente. Una vez obtenidas las fases deseadas se mezclaron durante 3 horas nuevamente en medio alcohólico, en un molino planetario en diferentes proporciones para conformar los compuestos xBNKT-(100-x)NCF $(x=80,70$ y 60$)$. El material compuesto se prensó y sinterizó a $1150{ }^{\circ} \mathrm{C}$ durante $2 \mathrm{~h}$, en atmósfera de aire, a una velocidad de calentamiento y enfriamiento de $5^{\circ} \mathrm{C} / \mathrm{min}$. Las pastillas sinterizadas se rectificaron con lijas de granulometría 320 y 600, para eliminar desviaciones en la composición generadas por la pérdida de alcalinos en la superficie y mantener una superficie plana para el contacto eléctrico.

\subsection{Caracterización del material}

Los análisis estructurales se realizaron a través de un equipo de patrones de difracción de rayos $\mathrm{X}$ (DRX) empleando un PANalytical, X'pert Pro con radiación $\mathrm{CuK} \alpha$. Los valores de 
densidad relativa se determinaron utilizando el método de Arquímedes, mientras que la caracterización microestructural se evaluó con un microscopio electrónico de barrido de emisión de campo (FE-SEM, Sigma, Zeiss, Alemania). La composición se analizó mediante espectroscopía de dispersión de energía de rayos X, (EDS, JEOL JXA 8230 Japón).

El comportamiento dieléctrico se analizó sobre los discos sinterizados, con electrodos de plata pintados sobre la superficie, a través de las curvas de permitividad real e imaginaria en el intervalo de frecuencias entre $100 \mathrm{~Hz}$ y $1 \mathrm{MHz}$ a temperatura ambiente, mediante el uso de un medidor LCR Hewlett Packard 4284. Para la polarización de los cerámicos, las muestras fueron dispuestas entre electrodos, en un baño de silicona a $100^{\circ} \mathrm{C}$ por 15 min y bajo la aplicación de una tensión CC de $3500 \mathrm{~V} / \mathrm{mm}$ (Vitrek V73, USA). La constante piezoeléctrica $\left(\mathrm{d}_{33}\right)$ se determinó utilizando un equipo medidor de $\mathrm{d}_{33}$ (YE2730A d 33 METER), sobre las muestras previamente polarizadas. Finalmente, la magnetización a temperatura ambiente en función del campo magnético se midió en un magnetómetro de muestra vibrante (Lakeshore 7300). Los ciclos fueron tomados entre $+15 \mathrm{y}-15 \mathrm{kOe}$.

\subsection{Coeficiente Magnetoeléctrico}

La medición dinámica del efecto $\mathrm{ME}$ se realizó bajo un campo magnético alterno de 20 Oe con una frecuencia de $1000 \mathrm{~Hz}$ colinealmente con el campo magnético continuo variable desde 0 a $15 \mathrm{kOe}$. Las muestras se colocaron sobre la celda que se observa en la Fig. 2. Se utilizaron alambres finos como contactos para reducir el ruido. Se colocó un amplificador de audio a la salida del generador de frecuencia del lock-in y se midió el voltaje de salida de la muestra en la entrada del lock-in. Los equipos (fuente de corriente continua y lock-in) se controlaron mediante una interfaz de usuario desarrollada en LabView. El coeficiente se obtuvo indirectamente a través del voltaje generado por el material, amplificado y limitado en banda, para mejorar la relación señal / ruido por el lock-in.

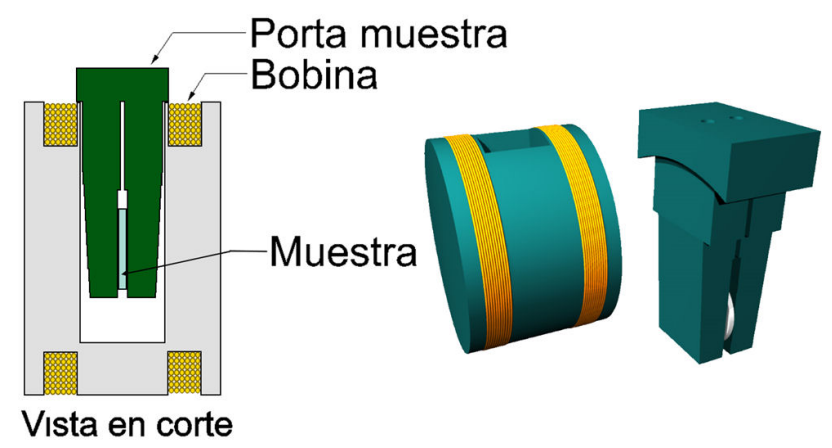

FIGURA 2. Celda de medición utilizada para soportar y sensar la muestra a temperatura ambiente.

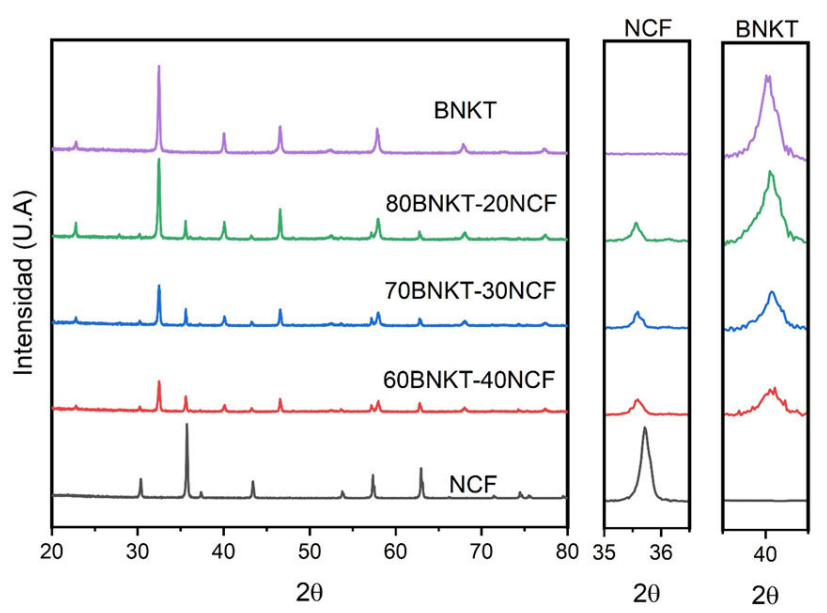

FIGURA 3. Diagramas de DRX de los compuestos xBNKT-(100$\mathrm{x}) \mathrm{NCF}(x=80,70, \mathrm{y} 60)$.

\section{Resultados y discusiones}

La Fig. 3 muestra los diagramas de DRX pertenecientes a las mezclas BNKT-NCF preparadas con diferentes relaciones BNKT-NCF (60-40, 70-30 y 80-20). En la parte superior e inferior de la misma figura, se presentan los diagramas del BNKT (perovskita) y NCF (espinela) obtenidos por reacción en estado sólido. En los diagramas de las mezclas se advierte un cambio en las intensidades de los picos pertenecientes a cada una de las fases. Al analizar el pico principal de la ferrita se advierte un corrimiento del mismo hacia menores ángulos a medida que aumenta la proporción de la fase BNKT. Esta variación indica que se produce una expansión de la celda, producto de la difusión de iones de radio más grande en sitios de iones más pequeños en el material NCF [9].

En la Fig. 4 se observan las imágenes de microscopía electrónica de barrido (MEB) de las mezclas sinterizadas a $1150^{\circ} \mathrm{C}$ por 5 horas. En ellas se aprecia un importante incremento en el tamaño de grano a medida que disminuye la cantidad de NCF. También se puede observar la existencia de una distribución bimodal de tamaños de grano y en la muestra de composición 70/30 se detecta claramente una tercera fase en forma de barras. Por ello, se realizó un estudio mediante EDS con el objetivo de identificar la composición de las distintas zonas.

En la Fig. 5 se presenta el mapeo EDS realizado a la muestra 80BNKT-20NCF. A fin de analizar la distribución de los distintos elementos dentro de la muestra, se establece como referencia en este trabajo el mapeo correspondiente al titanio. Del análisis de la distribución de elementos, es posible identificar la presencia de al menos tres fases a citar:

- Primera Zona: posee un elevado contenido de iones titanio y potasio, y una pequeña cantidad de iones $\mathrm{Fe}$, Ni y Co. Dado que en esta zona el contenido de bismuto se encuentra por debajo del límite de detección de la técnica, se puede asignar esta región a la formación de una fase secundaria de baja concentración cuya 

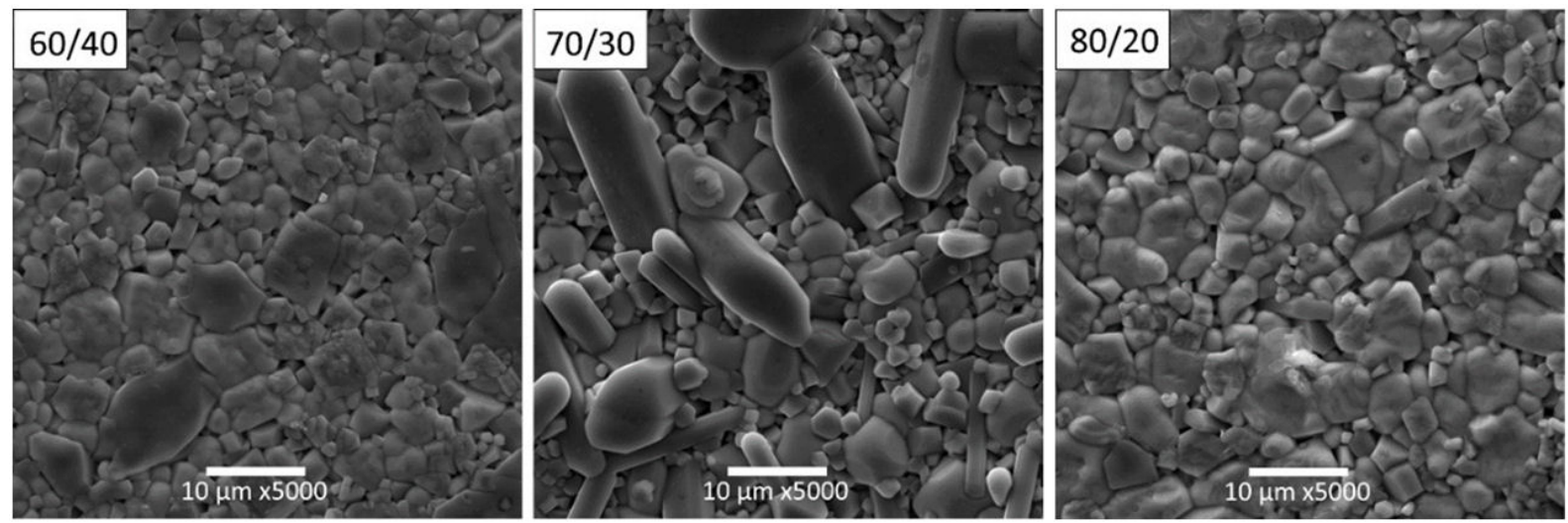

Figura 4. Imágenes MEB de las muestras xBNKT-(100-x)NCF $(x=80,70$ y 60).
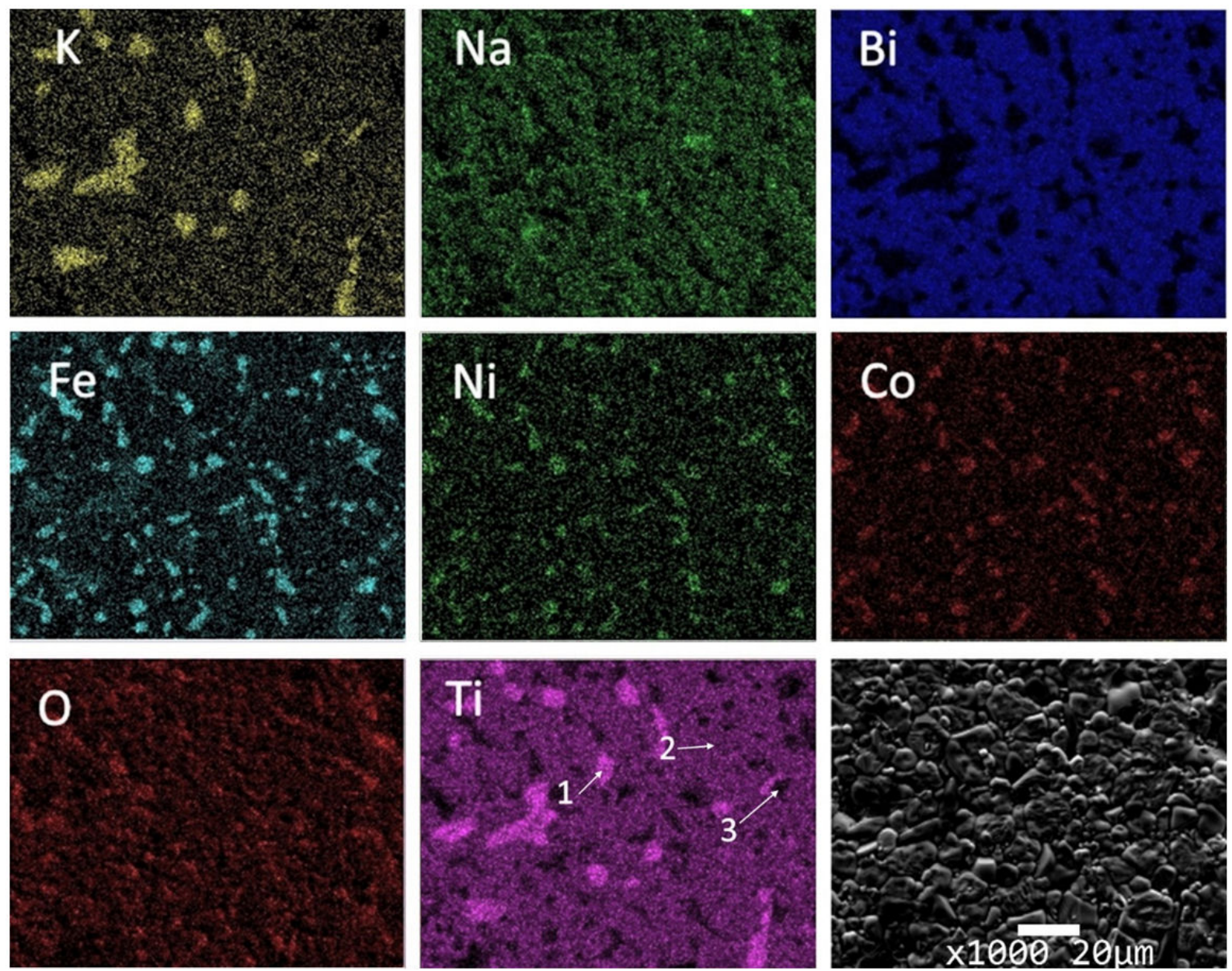

FIGURA 5. Mapeo por elementos de la muestra 80BNKT-20NCF.

composición se aleja de la correspondiente a las fases ferrita y piezoeléctrica [10-11].

- Segunda Zona: registra menor intensidad de Ti, denominada "matriz". Presenta una estequiometria muy similar a la del sistema BNKT, tal como se muestra en los patrones de difracción de rayos $\mathrm{X}$, con pequeños contenidos de $\mathrm{Fe}$, Co y $\mathrm{Ni}$.

- Tercera Zona: corresponde a las zonas negras en el mapeo del titanio, o zonas sin titanio. En los otros mapeos se observa la ausencia de bismuto y una alta concentración de $\mathrm{Fe}$, Ni y Co, tal como se esperaría para la fase NCF. Aqui se encuentran pequeñas cantidades de $\mathrm{K}$ y $\mathrm{Na}$. 
Estas tres zonas se observaron en todas las muestras analizadas. Lo anterior indica que, aunque las fases ferrita y piezoeléctrica se sintetizaron por separado, durante el proceso de sinterización se produjo la difusión de diferentes iones, lo cual desencadena una fase secundaria y el correspondiente cambio en las composiciones estequiométricas de las fases piezoeléctrica y ferrita. Además, esta segunda fase y el aumento de la cantidad de ferrita son responsables de la disminución del tamaño de grano de la matriz de BNKT.

La Tabla I muestra los valores de densidad, permitividad real, constante piezoeléctrica $\left(\mathrm{d}_{33}\right)$, magnetización de saturación (Ms) y coeficiente magnetoeléctrico $\left(\alpha^{*}\right)$ de compuestos de BNKT-NCF con diferentes relaciones de BNKT-NCF. Se observa que los valores de permitividad real, densidad y constante piezoeléctrica aumentan con el contenido de BNKT (fase piezoeléctrica), mientras que la magnetización de saturación disminuye con el contenido de BNKT. En este sentido, la concentración 70-30 presenta una relación más balanceada entre las propiedades piezoeléctricas y magnéticas, y genera el mejor valor de coeficiente magnetoeléctrico debido al acoplamiento entre las fases.

Finalmente, la Fig. 6 muestra la respuesta magnetoeléctrica en función de la frecuencia y de la relación entre BNKT y $\mathrm{NCF}$ determinada, a temperatura ambiente y a una frecuencia de $10 \mathrm{kHz}$, con la configuración mostrada en la Fig. 1. Al comparar la respuesta de los diferentes sistemas, se aprecia el desarrollo de una señal simétrica respecto a la polarizada del campo continuo. Este comportamiento y los valores encontrados de coeficiente magnetoeléctrico (Tabla I) son consistentes con los reportados para materiales multiferroicos similares [12-13]. Finalmente, los compuestos con una relación 70-30 mostraron una diferencia sustancial en la respuesta magnetoeléctrica debido al mejor acoplamiento entre las propiedades piezoeléctricas y magnéticas. Si se obser-

TABLA I. Valores de densidad $(\rho)$, permitividad real ( $\varepsilon^{\prime}$ a $\left.10 \mathrm{kHz}\right)$, constante piezoeléctrica $\left(\mathrm{d}_{33}\right)$, magnetización de saturación $(\mathrm{Ms})$, y coeficiente magnetoeléctrico $\left(\alpha^{*}\right)$ de compuestos BNKT-NCF.

\begin{tabular}{cccccc}
\hline BNKT-NCF & $\begin{array}{c}\rho \\
\left(\mathrm{g} / \mathrm{cm}^{3}\right)\end{array}$ & $\varepsilon^{\prime}$ & $\begin{array}{c}\mathrm{d}_{33} \\
(\mathrm{pC} / \mathrm{N})\end{array}$ & $\begin{array}{c}\mathrm{Ms} \\
(\mathrm{emu} / \mathrm{g})\end{array}$ & $\begin{array}{c}\alpha^{*} \\
(\mathrm{mV} / \mathrm{cmOe})\end{array}$ \\
\hline $60-40$ & $5.17 \pm 0.03$ & 287 & 10 & 22.9 & 0.69 \\
$70-30$ & $5.19 \pm 0.02$ & 417 & 24 & 17.7 & 4.81 \\
$80-20$ & $5.32 \pm 0.09$ & 507 & 28 & 9.8 & 2.30 \\
\hline
\end{tabular}
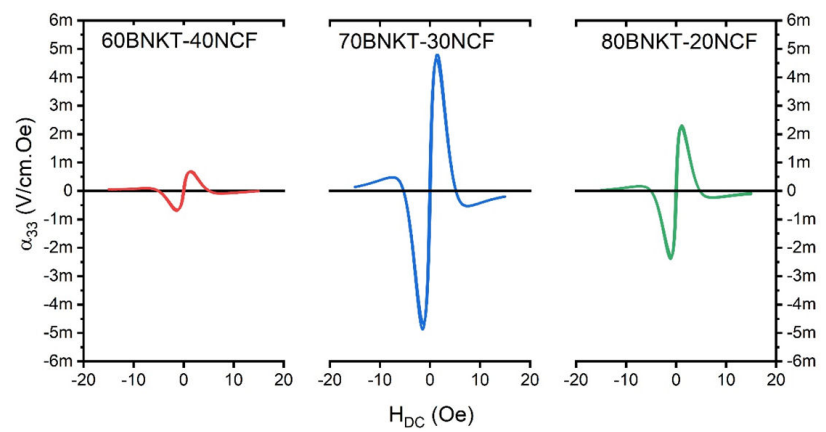

FIGURA 6. Variación del coeficiente magnetoeléctrico en función de la densidad del flujo magnético continuo a temperatura ambiente y a $1 \mathrm{kHz}$ correspondiente a compuestos de composiciones xBNKT-(100-x)NCF $(x=80,70$ y 60$)$.

va el comportamiento a altos campos, se detecta un cambio de signo de la respuesta magnetoeléctrica ligada a la respuesta magnetostrictiva tipica de las ferritas con presencia de Co y $\mathrm{Ni}$, dado que estas presentan un efecto de compresión luego de superar un valor de campo [14].

\section{Conclusiones}

En este trabajo se implementó un sistema para medir el coeficiente magnetoeléctrico $\left(\alpha^{*}\right)$ a temperatura ambiente a partir del método dinámico en materiales multiferroicos. El estudio se complementó con el análisis del comportamiento magnetoeléctrico de cerámicos compuestos libres de plomo tipo BNKT-NCF. Se observó que el coeficiente magnetoeléctrico de las pastillas cerámicas variaba con la relación de BNKT/NCF, lo cual arrojó valores optimos en las composiciones que presentaban un mejor balance entre las propiedades piezoeléctricas y magnéticas. En este sentido, se observó un comportamiento magnetostrictivo característico de las ferritas de Co y Ni.

\section{Agradecimientos}

Los autores agradecen a la Universidad Nacional de Mar del Plata, al Consejo Nacional de Investigaciones Científicas y Técnicas y a la Agencia Nacional de Promoción Científica y Tecnológica por el financiamiento recibido.
1. J. Camargo, L. Ramajo, F. Rubio-Marcos, M. Castro, $A d v$. Mat. Res., 975 (2014) 3-8. https : / / doi.org/10.4028/ WwW.scientific.net/AMR.975.3

2. K. Gil J. Gil, B. Cruz, A. Ramírez, M. Medina y J. Torres, J. Phys.: Conference Series. 687 (2016) 012090. https : //doi.org/10.1088/1742-6596/687/1/012090

3. C. W. Nan, M.I. Bichurin, S. Dong, D. Viehland, y G. Srini- vasan, J. App.Phys., 103 (2008) 031101. https://doi. org/10.1063/1.2836410

4. S. Liu et al., J. App. Phys., 122 (2017) 034103. https: //doi.org/10.1063/1.4994172

5. A. J. C. Buurma, G. R. Blake, T. T. M. Palstra, and U. Adem, Reference Module in Materials Science and Materials Engineering (2016). 
6. S. Mazumder, G. S. Bhattacharya, Mat. Res. Bulleting, 38 (2003) 303-310. https://doi.org/10.1016/ S0025-5408(02) 01048-6.

7. G. S. Bhattacharya, S. Mazumder, and S. Baisnab, Ind. J. Eng. Mat. Sci., 4 (2004) 185-188.

8. J. P. Rivera, Ferroelectrics 161 (1994) 147-165. https:// doi.org/10.1080/00150199408213364

9. J. Camargo, A. Prado Espinosa, F. Zabotto, L. Ramajo, and M. Castro, J. Alloys and Comp., doi.org/10.1016/j. jallcom.2020.154129

10. X. Meng, D. Wang, J. Liu, B. Lin, and Z. Fu, Sol. State Commun. 137 (2006) 146. https://doi.org/10.1016/j. SSC.2005.11.004
11. H. Zhang, J. Zhou, W. Chen, W. Yang, J. Shen, and C. Wu, J. Electron. Mater. 46 (2017) 6167-6174. https : / / doi . org/ 10.1007/s11664-017-5656-9

12. D. Shi, K. H. Lam, K. Li, Journals of Alloys and Compounds, 617 (2014) 485-490. https://doi.org/10.1016/j. jallcom.2014.08.016

13. V. R. Mudinepalli, S.-H. Song, J.-Q. Li, and B. S. Murty, J. Magn. Magnetic Mat., 386 (2015) 44-49. https://doi. org/10.1016/j.jmmm.2015.03.059

14. R. M. Bozorth, Elizabeth F. Tilden, and Albert J. Williams, Phys. Rev., 99 (1955) 1788-1798 https://doi.org/10. 1103/PhysRev.99.1788 
ELSEVIER LICENSE

TERMS AND CONDITIONS

May 12,2020

This Agreement between Dr. Javier Camargo ("You") and Elsevier ("Elsevier") consists of your license details and the terms and conditions provided by Elsevier and Copyright Clearance Center.

License Number

4826610502522

License date

May 12, 2020

Licensed Content

Publisher

Elsevier

Licensed Content

Publication

Journal of Alloys and Compounds

$\begin{array}{ll}\text { Licensed Content Title } & \begin{array}{l}\text { Magnetoelectric interactions in bismuth sodium-potassium } \\ \text { titanate-nickel cobalt ferrite lead-free composite ceramics }\end{array}\end{array}$

Licensed Content Author J. Camargo,A. Prado Espinosa,F. Zabotto,L. Ramajo,M. Castro

Licensed Content Date Jun 15, 2020

Licensed Content Volume 826

Licensed Content Issue n/a

Licensed Content Pages 1

Start Page 154129

End Page $\quad 0$

Type of Use reuse in a journal/magazine 
CONFIGURACIÓN Y MONTAJE DE INSTRUMENTO PARA LA CARACTERIZACIÓN MAGNETOELÉCTRICA...

$12 / 5 / 2020$

RightsLink Printable License

Requestor type academic/educational institute

Portion figures/tables/illustrations

Number of

figures/tables/illustrations 5

Format both print and electronic

Are you the author of this Yes

Elsevier article?

Will you be translating? Yes, without English rights

Number of languages $\quad 1$

Title of new article Configuración y Montaje de Instrumento para la Caracterización Magnetoeléctrica de Compuestos Cerámicos Multiferroicos

Lead author Javier Camargo

Title of targeted journal Revista Mexicana de Fisica

Publisher Siciedad Mexicana de Fisica

Expected publication date Jul 2020

Portions parts of figures $2,4,5$, and 10

Specific Languages Spanish

Dr. Javier Camargo

Colon 10400

Requestor Location

Mar del Plata, Buenos Aires 7600

Argentina

Attn: Dr. Javier Camargo

Publisher Tax ID

GB 494627212

Total

0.00 USD

https://s100.copyright.com/CustomerAdmin/PLF.jsp?ref=57c5867a-6a04-4025-b682-ffdd572c3981 
Terms and Conditions

\section{INTRODUCTION}

1. The publisher for this copyrighted material is Elsevier. By clicking "accept" in connection with completing this licensing transaction, you agree that the following terms and conditions apply to this transaction (along with the Billing and Payment terms and conditions established by Copyright Clearance Center, Inc. ("CCC"), at the time that you opened your Rightslink account and that are available at any time at http://myaccount.copyright.com).

\section{GENERAL TERMS}

2. Elsevier hereby grants you permission to reproduce the aforementioned material subject to the terms and conditions indicated.

3. Acknowledgement: If any part of the material to be used (for example, figures) has appeared in our publication with credit or acknowledgement to another source, permission must also be sought from that source. If such permission is not obtained then that material may not be included in your publication/copies. Suitable acknowledgement to the source must be made, either as a footnote or in a reference list at the end of your publication, as follows:

"Reprinted from Publication title, Vol/edition number, Author(s), Title of article / title of chapter, Pages No., Copyright (Year), with permission from Elsevier [OR APPLICABLE SOCIETY COPYRIGHT OWNER]." Also Lancet special credit - "Reprinted from The Lancet, Vol. number, Author(s), Title of article, Pages No., Copyright (Year), with permission from Elsevier."

4. Reproduction of this material is confined to the purpose and/or media for which permission is hereby given.

5. Altering/Modifying Material: Not Permitted. However figures and illustrations may be altered/adapted minimally to serve your work. Any other abbreviations, additions, deletions and/or any other alterations shall be made only with prior written authorization of Elsevier Ltd. (Please contact Elsevier at permissions@elsevier.com). No modifications can be made to any Lancet figures/tables and they must be reproduced in full.

6. If the permission fee for the requested use of our material is waived in this instance, please be advised that your future requests for Elsevier materials may attract a fee.

7. Reservation of Rights: Publisher reserves all rights not specifically granted in the combination of (i) the license details provided by you and accepted in the course of this licensing transaction, (ii) these terms and conditions and (iii) CCC's Billing and Payment terms and conditions.

8. License Contingent Upon Payment: While you may exercise the rights licensed immediately upon issuance of the license at the end of the licensing process for the transaction, provided that you have disclosed complete and accurate details of your proposed use, no license is finally effective unless and until full payment is received from you (either by publisher or by CCC) as provided in CCC's Billing and Payment terms and conditions. If full payment is not received on a timely basis, then any license preliminarily granted shall be deemed automatically revoked and shall be void as if never granted. Further, in the event that you breach any of these terms and conditions or any of CCC's Billing and Payment terms and conditions, the license is automatically revoked and shall be void as if never granted. Use of materials as described in a revoked license, as well as any use of the materials beyond the scope of an unrevoked license, may constitute copyright infringement 
and publisher reserves the right to take any and all action to protect its copyright in the materials.

9. Warranties: Publisher makes no representations or warranties with respect to the licensed material.

10. Indemnity: You hereby indemnify and agree to hold harmless publisher and CCC, and their respective officers, directors, employees and agents, from and against any and all claims arising out of your use of the licensed material other than as specifically authorized pursuant to this license.

11. No Transfer of License: This license is personal to you and may not be sublicensed, assigned, or transferred by you to any other person without publisher's written permission.

12. No Amendment Except in Writing: This license may not be amended except in a writing signed by both parties (or, in the case of publisher, by CCC on publisher's behalf).

13. Objection to Contrary Terms: Publisher hereby objects to any terms contained in any purchase order, acknowledgment, check endorsement or other writing prepared by you, which terms are inconsistent with these terms and conditions or CCC's Billing and Payment terms and conditions. These terms and conditions, together with CCC's Billing and Payment terms and conditions (which are incorporated herein), comprise the entire agreement between you and publisher (and CCC) concerning this licensing transaction. In the event of any conflict between your obligations established by these terms and conditions and those established by CCC's Billing and Payment terms and conditions, these terms and conditions shall control.

14. Revocation: Elsevier or Copyright Clearance Center may deny the permissions described in this License at their sole discretion, for any reason or no reason, with a full refund payable to you. Notice of such denial will be made using the contact information provided by you. Failure to receive such notice will not alter or invalidate the denial. In no event will Elsevier or Copyright Clearance Center be responsible or liable for any costs, expenses or damage incurred by you as a result of a denial of your permission request, other than a refund of the amount(s) paid by you to Elsevier and/or Copyright Clearance Center for denied permissions.

\section{LIMITED LICENSE}

The following terms and conditions apply only to specific license types:

15. Translation: This permission is granted for non-exclusive world English rights only unless your license was granted for translation rights. If you licensed translation rights you may only translate this content into the languages you requested. A professional translator must perform all translations and reproduce the content word for word preserving the integrity of the article.

16. Posting licensed content on any Website: The following terms and conditions apply as follows: Licensing material from an Elsevier journal: All content posted to the web site must maintain the copyright information line on the bottom of each image; A hyper-text must be included to the Homepage of the journal from which you are licensing at

http://www.sciencedirect.com/science/joumal/xxxxx or the Elsevier homepage for books at http://www.elsevier.com; Central Storage: This license does not include permission for a scanned version of the material to be stored in a central repository such as that provided by Heron/XanEdu.

Licensing material from an Elsevier book: A hyper-text link must be included to the Elsevier homepage at http://www.elsevier.com . All content posted to the web site must maintain the copyright information line on the bottom of each image. 
Posting licensed content on Electronic reserve: In addition to the above the following clauses are applicable: The web site must be password-protected and made available only to bona fide students registered on a relevant course. This permission is granted for 1 year only. You may obtain a new license for future website posting.

17. For journal authors: the following clauses are applicable in addition to the above:

\section{Preprints:}

A preprint is an author's own write-up of research results and analysis, it has not been peerreviewed, nor has it had any other value added to it by a publisher (such as formatting, copyright, technical enhancement etc.).

Authors can share their preprints anywhere at any time. Preprints should not be added to or enhanced in any way in order to appear more like, or to substitute for, the final versions of articles however authors can update their preprints on arXiv or RePEc with their Accepted Author Manuscript (see below).

If accepted for publication, we encourage authors to link from the preprint to their formal publication via its DOI. Millions of researchers have access to the formal publications on ScienceDirect, and so links will help users to find, access, cite and use the best available version. Please note that Cell Press, The Lancet and some society-owned have different preprint policies. Information on these policies is available on the journal homepage.

Accepted Author Manuscripts: An accepted author manuscript is the manuscript of an article that has been accepted for publication and which typically includes authorincorporated changes suggested during submission, peer review and editor-author communications.

Authors can share their accepted author manuscript:

- immediately

- via their non-commercial person homepage or blog

- by updating a preprint in arXiv or RePEc with the accepted manuscript

- via their research institute or institutional repository for internal institutional uses or as part of an invitation-only research collaboration work-group

- directly by providing copies to their students or to research collaborators for their personal use

- for private scholarly sharing as part of an invitation-only work group on commercial sites with which Elsevier has an agreement

- After the embargo period

○ via non-commercial hosting platforms such as their institutional repository

- via commercial sites with which Elsevier has an agreement

In all cases accepted manuscripts should:

- link to the formal publication via its DOI

- bear a CC-BY-NC-ND license - this is easy to do

- if aggregated with other manuscripts, for example in a repository or other site, be shared in alignment with our hosting policy not be added to or enhanced in any way to appear more like, or to substitute for, the published journal article.

Published journal article (JPA): A published journal article (PJA) is the definitive final record of published research that appears or will appear in the journal and embodies all value-adding publishing activities including peer review co-ordination, copy-editing, formatting, (if relevant) pagination and online enrichment. 
Policies for sharing publishing journal articles differ for subscription and gold open access articles:

Subscription Articles: If you are an author, please share a link to your article rather than the full-text. Millions of researchers have access to the formal publications on ScienceDirect, and so links will help your users to find, access, cite, and use the best available version.

Theses and dissertations which contain embedded PJAs as part of the formal submission can be posted publicly by the awarding institution with DOI links back to the formal publications on ScienceDirect.

If you are affiliated with a library that subscribes to ScienceDirect you have additional private sharing rights for others' research accessed under that agreement. This includes use for classroom teaching and internal training at the institution (including use in course packs and courseware programs), and inclusion of the article for grant funding purposes.

Gold Open Access Articles: May be shared according to the author-selected end-user license and should contain a CrossMark logo, the end user license, and a DOI link to the formal publication on ScienceDirect.

Please refer to Elsevier's posting.policy for further information.

18. For book authors the following clauses are applicable in addition to the above: Authors are permitted to place a brief summary of their work online only. You are not allowed to download and post the published electronic version of your chapter, nor may you scan the printed edition to create an electronic version. Posting to a repository: Authors are permitted to post a summary of their chapter only in their institution's repository.

19. Thesis/Dissertation: If your license is for use in a thesis/dissertation your thesis may be submitted to your institution in either print or electronic form. Should your thesis be published commercially, please reapply for permission. These requirements include permission for the Library and Archives of Canada to supply single copies, on demand, of the complete thesis and include permission for Proquest/UMI to supply single copies, on demand, of the complete thesis. Should your thesis be published commercially, please reapply for permission. Theses and dissertations which contain embedded PJAs as part of the formal submission can be posted publicly by the awarding institution with DOI links back to the formal publications on ScienceDirect.

\section{Elsevier Open Access Terms and Conditions}

You can publish open access with Elsevier in hundreds of open access journals or in nearly 2000 established subscription journals that support open access publishing. Permitted third party re-use of these open access articles is defined by the author's choice of Creative Commons user license. See our open access license policy. for more information.

Terms \& Conditions applicable to all Open Access articles published with Elsevier:

Any reuse of the article must not represent the author as endorsing the adaptation of the article nor should the article be modified in such a way as to damage the author's honour or reputation. If any changes have been made, such changes must be clearly indicated.

The author(s) must be appropriately credited and we ask that you include the end user license and a DOI link to the formal publication on ScienceDirect.

If any part of the material to be used (for example, figures) has appeared in our publication with credit or acknowledgement to another source it is the responsibility of the user to ensure their reuse complies with the terms and conditions determined by the rights holder. 
Additional Terms \& Conditions applicable to each Creative Commons user license:

CC BY: The CC-BY license allows users to copy, to create extracts, abstracts and new works from the Article, to alter and revise the Article and to make commercial use of the Article (including reuse and/or resale of the Article by commercial entities), provided the user gives appropriate credit (with a link to the formal publication through the relevant DOI), provides a link to the license, indicates if changes were made and the licensor is not represented as endorsing the use made of the work. The full details of the license are available at http://creativecommons.org/licenses/by/4.0.

CC BY NC SA: The CC BY-NC-SA license allows users to copy, to create extracts, abstracts and new works from the Article, to alter and revise the Article, provided this is not done for commercial purposes, and that the user gives appropriate credit (with a link to the formal publication through the relevant DOI), provides a link to the license, indicates if changes were made and the licensor is not represented as endorsing the use made of the work. Further, any new works must be made available on the same conditions. The full details of the license are available at http://creativecommons.org/licenses/by-nc-sa/4.0.

CC BY NC ND: The CC BY-NC-ND license allows users to copy and distribute the Article, provided this is not done for commercial purposes and further does not permit distribution of the Article if it is changed or edited in any way, and provided the user gives appropriate credit (with a link to the formal publication through the relevant DOI), provides a link to the license, and that the licensor is not represented as endorsing the use made of the work. The full details of the license are available at $\underline{\mathrm{htt}} \mathrm{p}: /$ creativecommons.org/licenses/by-nc-nd/4.0. Any commercial reuse of Open Access articles published with a CC BY NC SA or CC BY NC ND license requires permission from Elsevier and will be subject to a fee.

Commercial reuse includes:

- Associating advertising with the full text of the Article

- Charging fees for document delivery or access

- Article aggregation

- Systematic distribution via e-mail lists or share buttons

Posting or linking by commercial companies for use by customers of those companies.

20. Other Conditions:

v1.9

Questions? customercare@ copyright.com or +1-855-239-3415 (toll free in the US) or +1-978-646-2777. 